\title{
Comparación experimental de controladores PID clásico, PID no lineal y PID difuso para el caso de regulación
}

\author{
Luis Fidel Cerecero Natale ${ }^{1,3,4}$, Eduardo Campos Mercado ${ }^{2,3}$, \\ Julio Cesar Ramos Fernández ${ }^{3}$, Marco Antonio Marquéz Vera ${ }^{3}$, Irvin Arlin Chan $\mathrm{Ac}^{4}$ \\ ${ }^{1}$ Instituto Tecnológico de Cancún, Cancún, Quintana Roo, \\ México \\ ${ }^{2}$ CONACYT-UNISTMO, Tehuantepec, Oaxaca, \\ México \\ ${ }^{3}$ Universidad Politécnica de Pachuca, Zempoala, Hidalgo, \\ México \\ ${ }^{4}$ Universidad Tecnológica de la Riviera Maya, Quintana Roo, \\ México \\ lcerecero@itcancun.edu.mx, ecampos@conacyt.mx, \\ jramos@upp.edu.mx,marquez@upp.edu.mx, irvin.chan@utrivieramaya.edu.mx
}

Resumen. Este artículo presenta los resultados experimentales al comparar los Controladores PID Clásico, PID No Lineal y PID Difuso de Ganancias Programables usados en controladores de posición. Para la sintonización del PID clásico fue usado el segundo método de Ziegler-Nichols, el PID No Lineal fue diseñado basado en funciones de saturación con parámetros variables y el PID Difuso de Ganancias Programables fue propuesto por experiencia para minimizar el sobreimpulso. La serie de experimentos realizados mostraron que el PID Clásico presenta inestabilidad en los cambios de referencia abruptos, debido a esto se implementó el PID No Lineal con el que se logran seguir todas las referencias, presentando ligero sobreimpulso en las regiones que el control clásico se vuelve inestable, finalmente al implementar el PID Difuso se logró seguir todas las referencias sin presentar sobreimpulso y consumiendo menor cantidad de energía.

Palabras clave: control PID, difuso, control de posición.

\section{Experimental Comparison of Classical PID, Nonlinear PID and Fuzzy PID Controllers for the Case of Set-point Regulation}

Abstract. This article presents the experimental results when comparing the Controllers PID Classic, Nonlinear PID and Fuzzy Gain Scheduling PID used in position controllers, for the tuning of the Classical PID was used the second method 
of Ziegler-Nichols, the Nonlinear PID was designed based on saturation functions with variable parameters and the Fuzzy Gain Scheduling PID was Proposed by experience to minimize overshoot. The series of experiments conducted show that the Classic PID presents instability in the abrupt changes of the setpoint, due to this the non-linear PID is implemented with which they are achieved follow all the references, presenting light on impulse in the regions that the classic controller becomes unstable, finally when implementing the Fuzzy PID are managed to follow all the references without overshoot and consuming less amount of energy.

Keywords: PID control, fuzzy, position control.

\section{Introducción}

En este artículo se presenta un control de posición para un Motor de Corriente Directa de Imán Permanente (MCDIP) es un dispositivo electromecánico que convierte la energía eléctrica e corriente directa en energía mecánica. El primer MCDIP funcional y comercial fue inventado por Zenobe Grame en 1873. Dado que son confiables y operan en distintas potencias desde fracciones de Watt hasta Kilowatts, son usados en varios campos como el automotriz, aeroespacial, robótica, ingeniería biomédica, ingeniería pesada entre otras.

Diversas técnicas de control han sido propuestas para el MCDIP. Es un sistema no lineal, por lo tanto el tradicional controlador Proporcional Integral Derivativo (PID) sólo permite controlar sistemas que operan en áreas restringidas [7]. La plataforma experimental es un sistema de posicionamiento angular, donde se usa el control PID Clásico. El objetivo del control PID es mantener una posición de referencia a un valor asignado y ser capaz de aceptar de manera dinámica nuevos valores de referencia. Los controles de posición modernos requieren que los controladores sean capaces de hacer frente a variaciones de parámetros e incertidumbres del sistema [11].

La técnica de sintonización de Ziegler-Nichols es ampliamente conocida, como un método heurístico bastante exacto para determinar buenas configuraciones de controladores PI y PID para una amplia gama de procesos industriales comunes. Aunque los controladores PD generalmente no se recomiendan para procesos de carga cero, ya que suelen presentar grandes errores en estado estacionario. Los controladores convencionales no pueden dar buenos resultados para los procesos de carga cero, incluso el controlador PID sintonizado con Ziegler-Nichols no proporciona un rendimiento satisfactorio durante ese tiempo [12]. En tal situación el Controlador PID No Lineal puede mostrar un mejor rendimiento. Sin embargo, se vuelve más complejo para determinar los parámetros tradicionales del PID, no la eficiencia para el control del sistema.

Así que por esta razón, el Controlador PID No Lineal se calcula donde los parámetros dependen de la cantidad de error del sistema. El combinar el controlador clásico más el controlador no lineal, puede proporcionar grados de libertad adicionales para lograr un mejor rendimiento del sistema [1,2,5,9]. Las características de cambio de las funciones no lineales coinciden con el proceso de cambio ideal de los parámetros del control PID No Lineal, logrando buenos resultados de manera estática o dinámica y mejorando la calidad de control [8,3]. 
El artículo presente está organizado de las siguiente manera: en la sección 2 se describe brevemente la plataforma de experimentación y el modelo dinámico del MCDIP. La sintonización y experimentos del Controlador PID Clásico se presenta en la sección 3. El diseño y desempeño del Controlador PID No Lineal se describe en la sección 4. La estructura de reglas y pruebas del Controlador PID Difuso de Ganancias Programables se describen en la sección 5. Los resultados experimentales y conclusiones se discuten en la sección 6.

\section{Plataforma de experimentación}

La plataforma de experimentación es realmente importante, dado que se pueden evaluar los resultados teóricos. Actualmente existen varias opciones para adquirir plataformas experimentales para uso académico, utilizadas para probar diversas técnicas de control, el principal problema de estas son el alto costo y la arquitectura cerrada. En consecuencia fue desarrollada la plataforma de experimentación que se muestra en la Figura 1, donde se observa el diagrama de bloques de los dispositivos que la componen. La principal ventaja de la plataforma experimental es su bajo costo y su arquitectura abierta, ya que está basada en la tarjeta Arduino DUE, la que se encarga de envíar las señales al manejador de potencia tipo puente H L298N, un MCDIP con encoder para obtener la posición angular y una computadora.

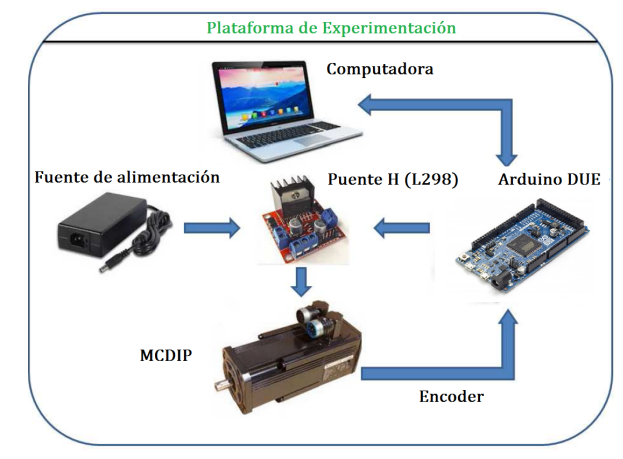

Fig. 1: Componentes de la plataforma de experimentación.

La computadora portátil es usada para programar el Arduino DUE y para graficar los resultados experimentales, con el propósito de observar el comportamiento del sistema. La tarjeta Arduino DUE es usada para leer los pulsos del sensor de posición, basado en un encoder óptico de 256 pulsos por revolución, procesar la estrategia de control usando un cristal oscilador de $84 \mathrm{MHz}$ y generar las señales moduladas en ancho de pulso (PWM) con una resolución de 12 bits, que a su vez polarizan al manejador de potencia tipo puente H modelo L298N. El MCDIP es de la marca PITTMAN, opera a $24 \mathrm{~V}$ y cuenta con el encoder óptico. 
Una plataforma muy similar para probar estrategias de control se muestra en [15], donde se logra implementar un controlador difuso para regular la velocidad de un MCDIP basado en la tarjeta electrónica Arduino DUE. Ahora, el modelo matemático de un MCDIP se puede escribir de la siguiente manera:

$$
J \ddot{\theta}+B \dot{\theta}=U,
$$

donde $U$ es el par torsional generado por la entrada de control, $J$ representa el momento polar de inercia, $B$ es el coeficiente de fricción viscosa, $\dot{\theta}$ y $\ddot{\theta}$ son la velocidad y aceleración angular respectivamente.

De la ecuación (1) se puede obtener la función de transferencia que relaciona la posición angular sobre la entrada de control, esto es:

$$
\frac{\theta(s)}{U(s)}=\frac{1}{J s^{2}+B s},
$$

Es importante darse cuenta que $J$ y $B$ son parámetros del MCDIP, que pueden ser identificados para usar una estrategia de control basada en el modelo matemático; pero en este artículo el interés se centra en trabajar con el modelo libre para establecer una estrategia de control.

El principal objetivo de los experimentos, es evaluar el comportamiento del sistema en lazo cerrado, es de suma importancia comentar que cada experimento tiene una duración de 15 segundos. Para evaluar el desempeño de los Controladores PID Clásico, PID No Lineal y el PID Difuso de Ganancias Programables, se establencen cambios en la referencia angular deseada $\theta_{d}$ en función del tiempo, cada 3 segundos como se muestra en la siguiente ecuación.

$$
\theta_{d}(t)=\left\{\begin{array}{ccl}
20^{\circ} & \text { si } & t \leq 3, \\
0^{\circ} & \text { si } & 3<t \leq 6, \\
90^{\circ} & \text { si } & 6<t \leq 9, \\
-60^{\circ} & \text { si } & 9<t \leq 12, \\
200^{\circ} & \text { si } & 12<t \leq 15 .
\end{array}\right.
$$

\section{Controlador PID clásico}

La técnica usada para sintonizar el controlador PID Clásico (PID-C), es el segundo método de Ziegler-Nichols [13]. Para este método es necesario usar un controlador proporcional y encontar el valor de la ganancia que genere oscilaciones sostenidas en el sistema. Las ganancias $k_{p}, k_{i}$ y $k_{d}$ son determinadas usando las ecuaciones (4), (5) y (6), que están en función de la la ganancia crítica de oscilación $K_{c r}=100$ y el periodo crítico $P_{c r}=0.11 s$, obtenidos por $\theta_{d}=0$, esto es:

$$
\begin{array}{cc}
k_{p}=0.6 \cdot K_{c r}, & \\
t_{i}=0.5 \cdot P_{c r}, & k_{i}=k_{p} / t_{i}, \\
t_{d}=0.125 \cdot P_{c r}, & k_{d}=k_{p} \cdot t_{d} .
\end{array}
$$


Los valores usados en el sistema de control en lazo cerrado son $k_{p}=60 k_{i}=$ 1090.9 y $k_{d}=0.8250$. El resultado experimental del sistema de control en lazo cerrado se muestra en la Figura 2, donde se utiliza el Controlador PID-C, por otra parte en la Figura 3 se muestra la señal de control $u$. Es de suma importancia hacer notar que el sistema presenta inestabilidad. Dado que la oscilación del sistema es muy grande no se logra apreciar el seguimiento de las referencias de posición, para esto se realizó un corte de la gráfica en los primeros 9 segundos del experimento, con lo que se puede apreciar la respuesta en regulación de posición de las primeras 3 referencias usando el Controlador PID-C, como se muestra en la Figura 4 y en la Figura 5 se puede ver el comportamiento de la variable de control $u$. De lo que se puede concluir que las últimas dos $\theta_{d}$ están fuera de la zona de operación de la caracterización realizada para el Controlador PID-C. La manera en la que se realiza la derivada del error, es por medio del Filtro Alpha-Beta mostrado [10] sin utilizar el término Gamma, donde se pueden usar los parámetros unitarios y equivale al método de derivación de Euler.

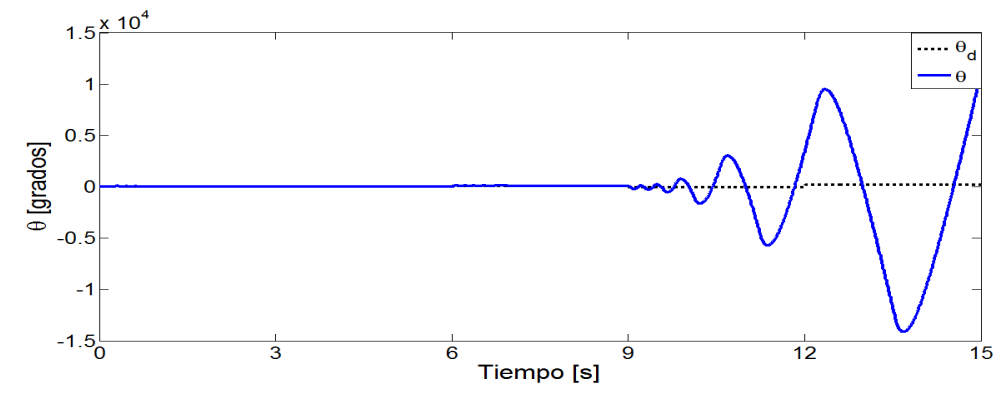

Fig. 2: Respuesta en regulación de posición, comportamiento inestable.

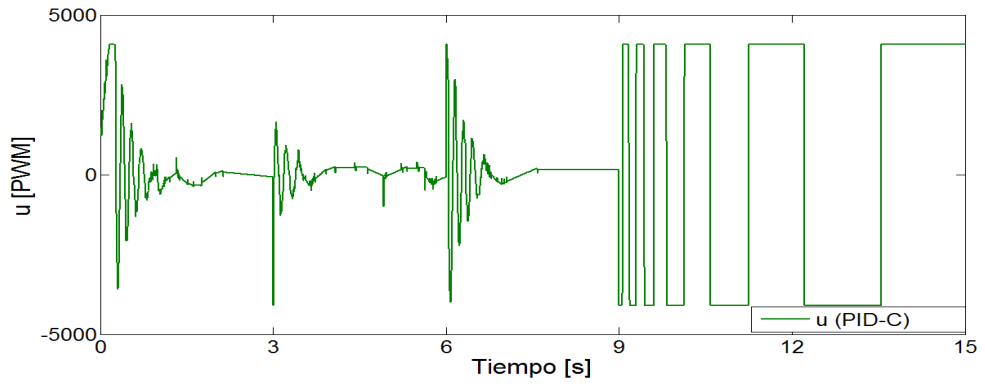

Fig. 3: Respuesta de la variable de control $u$, comportamiento inestable. 
Luis Fidel Cerecero Natale, Eduardo Campos Mercado, Julio Cesar Ramos Fernández, et al.

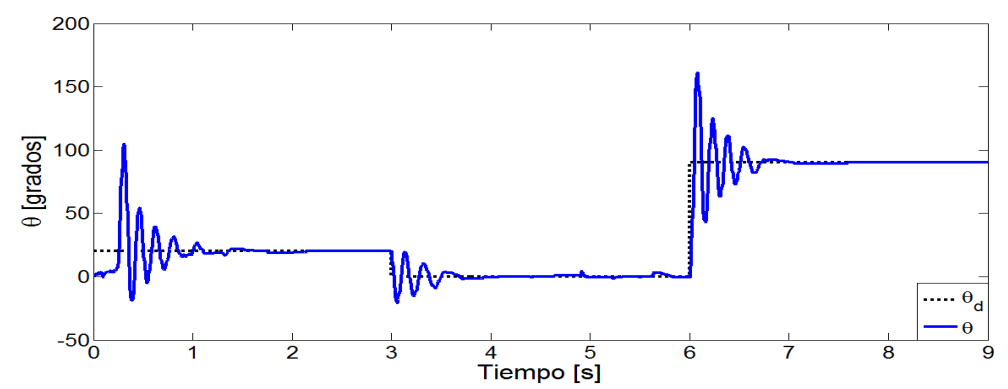

Fig. 4: Respuesta en regulación de posición, comportamiento inestable (9 segundos).

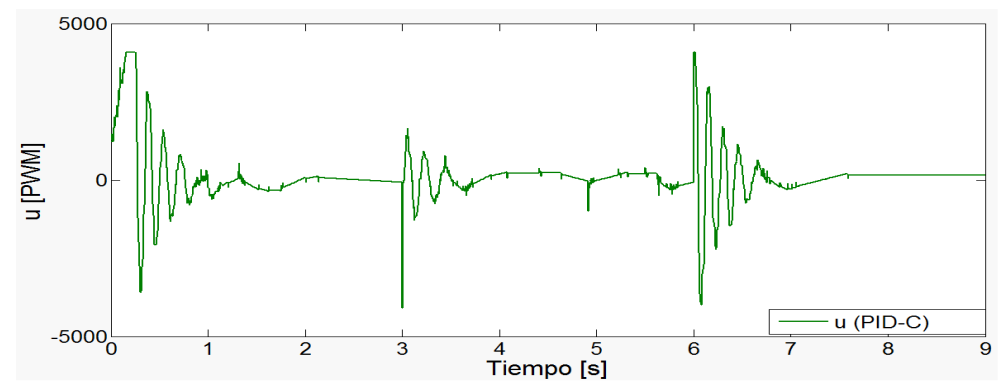

Fig. 5: Respuesta de la variable de control $u$, comportamiento inestable (9 segundos).

\section{Controlador PID no lineal}

En esta sección se presenta, un Controlador PID No Lineal (PID-NL) basado en funciones de saturación con parámetros variables, que es propuesto para regular la posición angular del MCDIP. Las funciones de saturación propuestas que son usadas para el controlador, son definidas por el conjunto de ecuaciones (7).

$$
\sigma_{\bar{b}}(\varphi)=\left\{\begin{array}{ccl}
\bar{b} & \text { si } & \varphi>\bar{b} \\
\varphi & \text { si } & |\varphi| \leq \bar{b} \\
-\bar{b} & \text { si } & \varphi<-\bar{b}
\end{array}\right.
$$

donde $\bar{b}$ es una constante positiva y $\varphi$ representa una función. En este caso, los términos a los que la saturación debe ser aplicada, son el error y su primera derivada respecto al tiempo.

Entonces, si se introduce la función de saturación dentro del Controlador PID-C se obtiene el Controlador PID-NL que se representa en la ecuación (8).

$$
U_{P I D-N L}=\sigma_{\bar{b}_{p}}\left(k_{p} e(t)\right)+\sigma_{\bar{b}_{d}}\left(k_{d} \frac{d e(t)}{d t}\right)+\sigma_{\bar{b}_{I}}\left(k_{I} \int_{0}^{t} e(\tau) d \tau\right),
$$

donde $k_{p}, k_{d}, k_{I} \in \mathbb{R}$ son definidos positivos, $e(t)=\theta_{d}(t)-\theta(t)$ representa el error. 
La ecuación (8) puede ser reescrita en su manera compacta usando la ecuación (9).

$$
U_{P I D-N L}=\sum_{i=1}^{3} u_{i} \quad \forall i=1,2,3,
$$

donde $u_{i}=\sigma_{\overline{b_{i}}}\left(k_{i} h_{i}\right)$ representa la función de saturación, con $\overline{b_{1}}=\overline{b_{p}}, \overline{b_{2}}=\overline{b_{d}}$, $\overline{b_{3}}=\overline{b_{I}}, k_{1}=k_{p}, k_{2}=k_{d}, k_{3}=k_{I} ; h_{1}=e(t), h_{2}=\frac{d e(t)}{d t}$ y $h_{3}=\int_{0}^{t} e(\tau) d \tau$. Entonces, para la ecuación (7) $u_{i}$ puede ser reescrita como:

$$
u_{i}=\left\{\begin{array}{ccl}
\bar{b}_{i} & \text { si } & k_{i} h_{i}>\bar{b}_{i} \\
k_{i} h_{i} & \text { si } & \left|k_{i} h_{i}\right| \leq \bar{b}_{i} \\
-\bar{b}_{i} & \text { si } & k_{i} h_{i}<-\bar{b}_{i}
\end{array}\right.
$$

De la ecuación (10) se puede notar que:

$$
\left|k_{i} h_{i}\right| \leq \bar{b}_{i}
$$

puede ser reescrita como: $\left|h_{i}\right| \leq \bar{b}_{i} / k_{i}$. En ese momento, se define:

$$
d_{i}:=\bar{b}_{i} / k_{i}
$$

En consecuencia se puede reescibir la ecuación (10) en términos de $d_{i}$, como se muestra a continuación:

$$
u_{i}=\left\{\begin{array}{ccl}
\bar{b}_{i} & \text { si } & h_{i}>d_{i}, \\
k_{i} h_{i} & \text { si } & \left|h_{i}\right| \leq d_{i}, \\
-\bar{b}_{i} & \text { si } & h_{i}<-d_{i} .
\end{array}\right.
$$

Ahora, la ecuación (13) puede ser simplificada como:

$$
u_{i}=\left\{\begin{array}{ccc}
\operatorname{sign}\left(h_{i}\right) \bar{b}_{i} & \text { si } & \left|h_{i}\right|>d_{i}, \\
\bar{b}_{i} d_{i}^{-1} h_{i} & \text { si } & \left|h_{i}\right| \leq d_{i} .
\end{array}\right.
$$

y se puede observar que con esta representación los parámetros de sintonización del controlador son $b_{i}$ y $d_{i}, \forall i=1,2$. Además, tomando en consideración:

$$
\operatorname{sign}\left(h_{i}\right) \bar{b}_{i}=h_{i} \operatorname{sign}\left(h_{i}\right) \bar{b}_{i} h_{i}^{-1},
$$

que puede simplificarse como:

$$
\operatorname{sign}\left(h_{i}\right) \bar{b}_{i}=\left|h_{i}\right| \bar{b}_{i} h_{i}^{-1}
$$

y se tiene que $\left|h_{i}\right| h_{i}^{-1}=\left|h_{i}\right|^{-1} h_{i}$, entonces la ecuación (14) puede ser reescrita como:

$$
u_{i}=\left\{\begin{array}{ccc}
\bar{b}_{i}\left|h_{i}\right|^{-1} h_{i} & \text { si } & \left|h_{i}\right|>d_{i} \\
\bar{b}_{i} d_{i}^{-1} h_{i} & \text { si } & \left|h_{i}\right| \leq d_{i}
\end{array}\right.
$$

Consecuentemente, la ley de control (9) puede ser expresada como:

$$
U_{P I D-N L}=u_{1}+u_{2}+u_{3},
$$


entonces

$$
U_{P I D-N L}=k_{p}(\cdot) e(t)+k_{d}(\cdot) \frac{d e(t)}{d t}+k_{I}(\cdot) \int_{0}^{t} e(\tau) d \tau
$$

con:

$$
\begin{gathered}
k_{p}(\cdot)=\left\{\begin{array}{cll}
\bar{b}_{p}|e(t)|^{-1} & \text { si } & |e(t)|>d_{p}, \\
\bar{b}_{p} d_{p}^{-1} & \text { si } & |e(t)| \leq d_{p},
\end{array}\right. \\
k_{d}(\cdot)=\left\{\begin{array}{cll}
\bar{b}_{d}\left|\frac{d e(t)}{d t}\right|^{-1} & \text { si } & \left|\frac{d e(t)}{d t}\right|>d_{d}, \\
\bar{b}_{d} d_{d}^{-1} & \text { si } & \left|\frac{d e(t)}{d t}\right| \leq d_{d},
\end{array}\right. \\
k_{I}(\cdot)=\left\{\begin{array}{ccc}
\bar{b}_{I}\left|\int_{0}^{t} e(\tau) d \tau\right|^{-1} & \text { si } & \left|\int_{0}^{t} e(\tau) d \tau\right|>d_{I}, \\
\bar{b}_{I} d_{I}^{-1} & \text { si } & \left|\int_{0}^{t} e(\tau) d \tau\right| \leq d_{I} .
\end{array}\right.
\end{gathered}
$$

La ventaja de esta formulación es que el par torsional es limitado por los parámetros $\bar{b}_{p}, \bar{b}_{d}$ y $\bar{b}_{I}$. Por lo tanto, se asegura la limitación de la entrada de control. Sin embargo, en algunos casos se puede requerir un par torsional ligeramente mayor para corregir los errores del sistema, es por eso que se propone el valor de saturación $\bar{b}_{i}$ de la ecuación (17) puede cambiar a:

$$
\bar{b}_{i}=b_{i}\left|h_{i}\right|^{\mu_{i}} \quad \text { si, } \quad\left|h_{i}\right|>d_{i}
$$

$\mathrm{y}$

$$
\bar{b}_{i}=b_{i}\left|d_{i}\right|^{\mu_{i}} \quad \text { si } \quad\left|h_{i}\right| \leq d_{i}
$$

con $b_{i}$ una constante positiva y $\mu_{i} \in[0,1]$.

Ahora, introduciendo las ecuaciones (23) y (24) dentro de (17), se obtiene:

$$
\begin{aligned}
& u_{i}=\left\{\begin{array}{ccc}
b_{i}\left|h_{i}\right|^{\mu_{i}}\left|h_{i}\right|^{-1} h_{i} & \text { si } & \left|h_{i}\right|>d_{i}, \\
b_{i}\left|d_{i}\right|^{\mu_{i}} d_{i}^{-1} h_{i} & \text { si } & \left|h_{i}\right| \leq d_{i},
\end{array}\right. \\
& \forall i=1,2,3 \text { у } \mu_{i} \in[0,1] .
\end{aligned}
$$

Los gráficos de la función para diferentes parámetros de $\mu_{i}$ se muestran en la Figura 6.

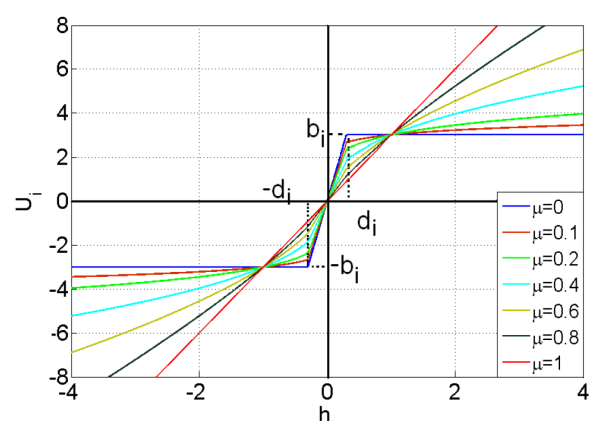

Fig. 6: Función de saturación con diferentes valores del parámetro $\mu$. 
Consecuentemente, la ley de control PID-NL basado en funciones de saturación con parámetros variables puede ser expresado como:

$$
U_{P I D-N L}=k_{p}(\cdot) e(t)+k_{d}(\cdot) \frac{d e(t)}{d t}+k_{I}(\cdot) \int_{0}^{t} e(\tau) d \tau
$$

con:

$$
\begin{gathered}
k_{p}(\cdot)=\left\{\begin{array}{ccl}
b_{p}|e(t)|^{\left(\mu_{p}-1\right)} & \text { si } & |e(t)|>d_{p}, \\
b_{p} d_{p}^{\left(\mu_{p}-1\right)} & \text { si } & |e(t)| \leq d_{p},
\end{array}\right. \\
k_{d}(\cdot)=\left\{\begin{array}{ccc}
b_{d}\left|\frac{d e(t)}{d t}\right|^{\left(\mu_{d}-1\right)} & \text { si } & \left|\frac{d e(t)}{d t}\right|>d_{d}, \\
b_{d} d_{d}^{\left(\mu_{d}-1\right)} & \text { si } & \left|\frac{d e(t)}{d t}\right| \leq d_{d},
\end{array}\right. \\
k_{I}(\cdot)=\left\{\begin{array}{ccc}
b_{I}\left|\int_{0}^{t} e(\tau) d \tau\right|^{\left(\mu_{I}-1\right)} & \text { si } & \left|\int_{0}^{t} e(\tau) d \tau\right|>d_{I}, \\
b_{I} d_{I}^{\left(\mu_{I}-1\right)} & \text { si } & \left|\int_{0}^{t} e(\tau) d \tau\right| \leq d_{I},
\end{array}\right. \\
\forall \mu_{p}, \mu_{d}, \mu_{I} \in[0,1] .
\end{gathered}
$$

De la Figura 6 se puede notar que, si $\mu_{p}=\mu_{d}=\mu_{I}=1$, el Controlador PID-NL que está dado por la ecuación (26) se degenera el controlador PID lineal. Además, si $\mu_{p}=\mu_{d}=\mu_{I}=0$, se obtiene el caso de una saturación constante. Para resumir, se puede concluir que el controlador PID lineal y el Controlador PID-NL con una función de saturación simple, definido por la ecuación (7), son casos particulares del controlador propuesto.

La sintonización del Controlador PID-NL fue determinado de manera experimental hasta que se logró un desempeño aceptable desde un punto de vista práctico, obteniendo los valores: $d_{p}=0.8, \mu_{p}=0.1, b_{p}=300 ; d_{d}=3, \mu_{d}=0.1, b_{d}=4 ; d_{I}=0.1$, $\mu_{I}=0, b_{I}=10$.

La Figura 7 muestra la respuesta de regulación del sistema de lazo cerrado usando el Controlador PID-NL propuesto, que viene dada por la ecuación (26). La Figura 8, es la respuesta en regulación de posición de la señal de control $u$ del Controlador PID-NL. Cabe resaltar que el sistema se muestra estable durante todo el experimento a diferencia del Controlador PID-C, otra característica importante es la suavidad que presenta al adaptarse a los cambios de referencia abruptos.

\section{Controlador PID difuso de ganancias programables}

En esta sección se presenta, el Controlador PID Difuso de Ganancias Programables (PID-DGP) para regular la posición angular del MCDIP, la estructura es muy similar a la que se logró en [4], pero la principal diferencia es que las reglas fueron pensadas para regular posiciones de referencia constante ante variaciones en la carga y en el trabajo actual están diseñadas para regular la posición ante cambios abruptos de referencias variables. En los controladores de velocidad difuso adaptativo y autosintonizable de $[6,16,14]$ presentan una estrucutra muy similar en cuanto a las variables utilizadas para entradas y salidas. Para la implementación de este controlador difuso se utiliza una estructura de Múltiples Entradas y Múltiples Salidas (MIMO) son definidas dos entradas: 
Luis Fidel Cerecero Natale, Eduardo Campos Mercado, Julio Cesar Ramos Fernández, et al.

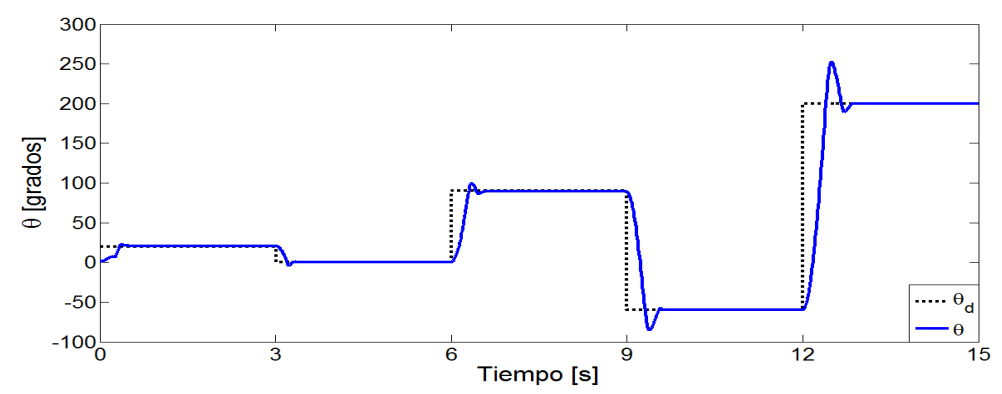

Fig. 7: Respuesta en regulación de posición.

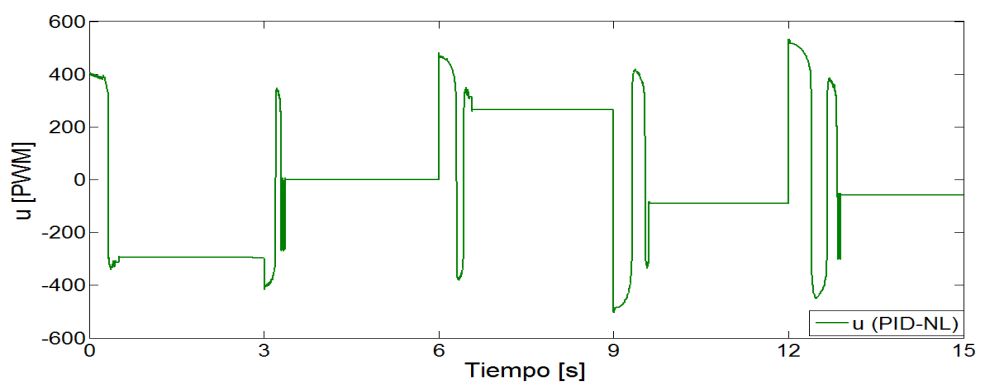

Fig. 8: Respuesta de la variable de control $u$.

el error $e$ y su primera derivada respecto del tiempo $\dot{e}$; y tres salidas: las ganancias difusas proporcional $K_{p F}$, integral $K_{i F}$ y derivativa $K_{d F}$. La Figura 9 representa el controlador PID-DGP, donde claramente se aprecia que las ganancias difusas afectan a las ganancias de un controlador PID-C, que para este caso en particular, las ganancias clásicas tienen asignados los valores $K_{p C}=90, K_{i C}=2 \mathrm{y} K_{d C}=3$, que fueron determinados de manera experimental de acuerdo al desempeño del sistema.

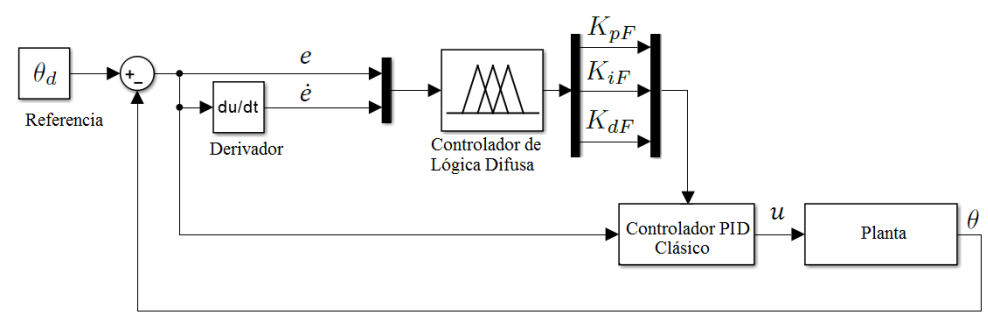

Fig. 9: Diagrama a bloques del sistema de control PID Difuso de Ganancias Programables. 
Las entradas $e$ y $\dot{e}$, son definidas por tres particiones difusas estáticas tipo Gaussianas, que son mostradas en la Figura 10, representa los valores de las funciones de pertenencia al instante dentro del ciclo de control. La estructura de reglas del controlador difuso se representan con la ecuación (30) el número de reglas por la ecuación (31) y el mecanismo de inferencia por la ecuación (32).

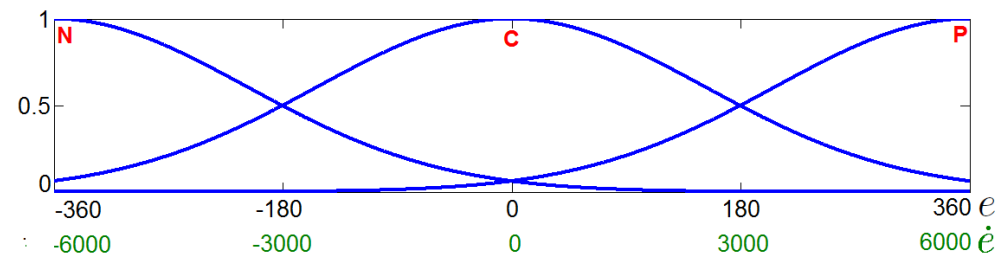

Fig. 10: Conjuntos difusos tipo Gaussianos para $e$ y $\dot{e}$.

$$
\begin{gathered}
R_{j} \text { : si e es } A_{j} \text { y è es } B_{j} \text { entonces } K_{p F(j)}=a_{j} y K_{i F(j)}=b_{j} y K_{d F(j)}=c_{j}, \\
R=\prod_{j=1}^{n} x_{j}, \\
\beta=\left(\mu_{e} \cdot \mu_{\dot{e}}\right) .
\end{gathered}
$$

En la Figura 11 se muestran las superficies de control no lineal para las ganancias $K_{p F}, K_{i F}$ y $K_{d F}$, básicamente es la representación tridimensional de las 9 reglas planteadas.

En la Tabla 1 se muestran las 9 reglas del controlador de lógica difusa para las entradas $e$ y $\dot{e}$ que corresponden a los valores constantes de los consecuentes lineales Singleton de orden cero, que se relacionan de manera directa con cada una de las ganancias, proporcional, integral y derivativa difusas que son acotadas $K_{p F}, K_{i F}$ y $K_{d} F \in[0,1]$.

La Figura 12 muestra la respuesta de regulación del sistema en lazo cerrado usando el Controlador PID-DGP, a diferencia del PID-NL aquí se logran seguir las referencias sin presentar sobreimpulso, sin importar que tan abrupto sea el cambio entre cada referencia asignada. La Figura 13, es la respuesta en regulación de posición de la señal de control $u$ del Controlador PID-DGP, donde se puede ver un comportamiento muy suave, que claramente consume mucho menor cantidad de energía en comparación con los controladores PID-C y PID-NL. Por último en la Figura 14 se muestra la variación de las ganancias programables difusas, que se generan al realizar el producto de las ganancias del controlador difuso con las ganancias del controlador clásico, como se muestra en la ecuación (33) cabe mencionar que la actividad de estas se hace más pronunciada a medida que los cambios de referencia son más abruptos. Otra característica que se puede notar de manera evidente, es el minúsculo error en estado estacionario debido a la atenuación paulatina de la ganancia $K_{i F}$ a medida que el error tiende a cero:

$$
K_{p}=K_{p F} \cdot K_{p C} \quad K_{i}=K_{i F} \cdot K_{i C} \quad K_{d}=K_{d F} \cdot K_{d C} .
$$


Luis Fidel Cerecero Natale, Eduardo Campos Mercado, Julio Cesar Ramos Fernández, et al.
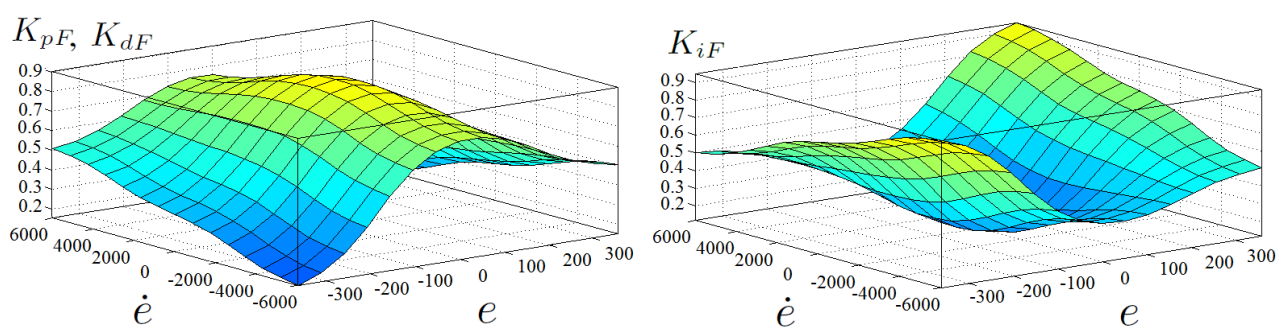

Fig. 11: Superficies de control no lineal para las ganancias difusas $K_{p F}, K_{i F}$ y $K_{d F}$.

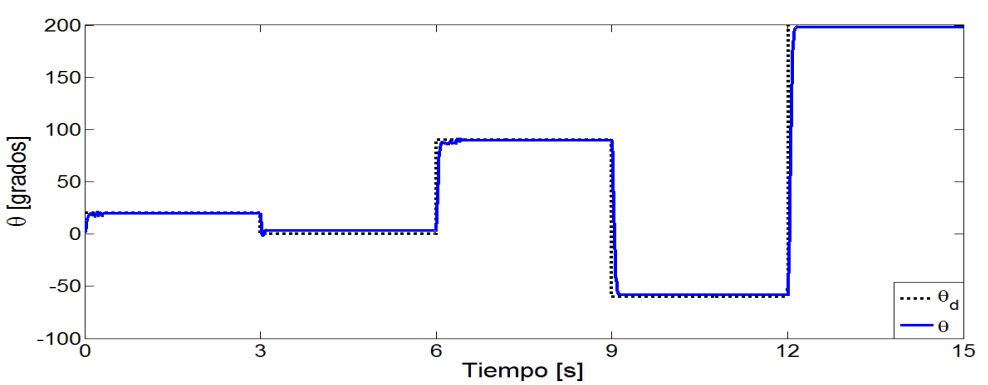

Fig. 12: Respuesta en regulación de posición.

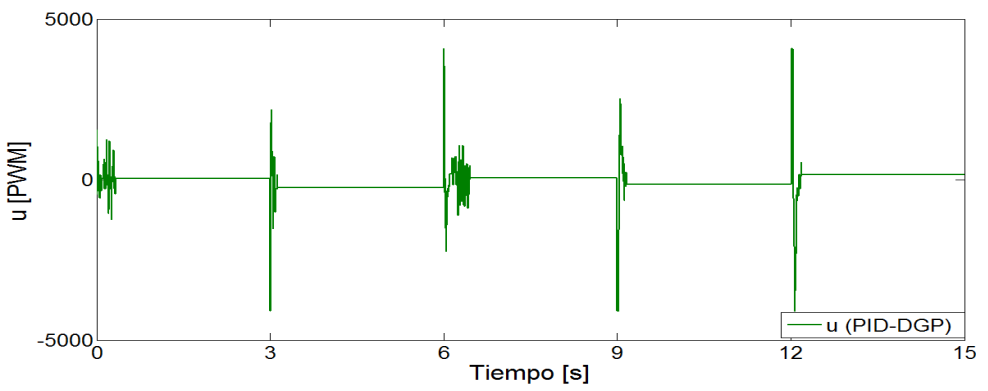

Fig. 13: Respuesta en regulación de posición de la variable de control $u$.

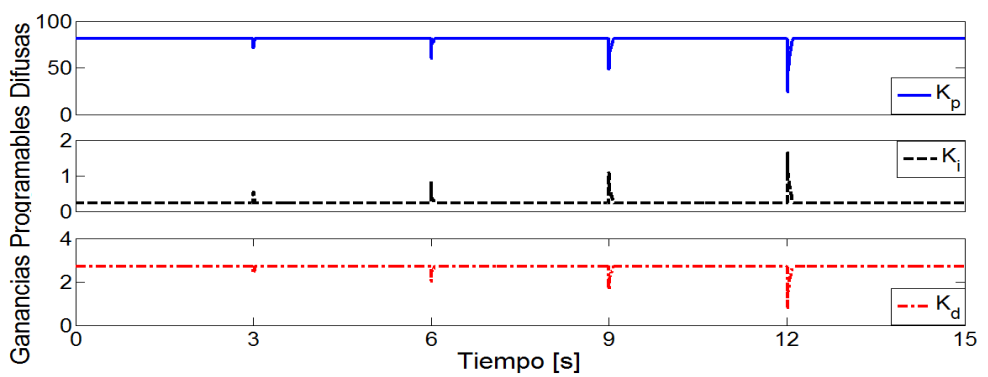

Fig. 14: Respuesta en regulación de posición (ganancias programables difusas $K_{p}, K_{i}$ y $K_{d}$ ). 
Comparación experimental de controladores PID clásico, PID no lineal y PID difuso ...

Tabla 1: Reglas difusas de sintonización para las ganancias $K_{p F}, K_{i F}$ y $K_{d F}$.

\begin{tabular}{|c|c|c|c|}
\hline \multicolumn{4}{|c|}{$e \dot{e} K_{p F} K_{i F} K_{d F}$} \\
\hline $\mathrm{N} \mathrm{N}$ & 0.1 & 1.0 & 0.1 \\
\hline $\mathrm{N} \mathrm{C}$ & 0.3 & 0.8 & 0.3 \\
\hline N P & 0.5 & 0.5 & 0.5 \\
\hline $\mathrm{C} \mathrm{N}$ & 0.8 & 0.3 & 0.8 \\
\hline $\mathrm{C} \mathrm{C}$ & 1.0 & 0.0 & 1.0 \\
\hline C P & 0.8 & 0.3 & 0.8 \\
\hline $\mathrm{P} \mathrm{N}$ & 0.5 & 0.5 & 0.5 \\
\hline $\mathrm{P} C$ & 0.3 & 0.8 & 0.3 \\
\hline $\mathrm{P} P$ & 0.1 & 1.0 & 0.1 \\
\hline
\end{tabular}

\section{Resultados experimentales}

Para evaluar el desempeño del sistema en lazo cerrado se cálcula la integral del error absoluto (IAE), expresada mediante la ecuación (34):

$$
I A E=\int_{0}^{T}|e(t)| d t .
$$

Una forma de conocer la energía consumida durante los experimentos es calculando la integral del valor ablsoluto de la entrada de control (E), como se muestra en la ecuación (35):

$$
E=\int_{0}^{t}|U(\tau)| d \tau
$$

En la Tabla 2 se muestra la comparación de la respuesta de los controladores para el caso de regulación de posición, la última columna (S/s) representa la cantidad de muestras por segundo de cada controlador. Cabe mencionar que para lograr una comparación precisa fue necesario utilizar la herramienta timeseries de MATLAB, dado que la cantidad de muestras por segundo difiere en cada controlador y por ende el número de datos del vector.

\begin{tabular}{|c|c|}
\hline Controlador & E S/s \\
\hline PID-C & 69433007667500187 \\
\hline PID-NL & 38810793580241 \\
\hline PID-DGP & 769100203 \\
\hline
\end{tabular}

Tabla 2: Comparación de la respuesta de los controladores para el caso de regulación. 


\section{Conclusiones}

Con los resultados experimentales se logró realizar el estudio comparativo de las estrategias de control propuestas, evaluando principalmente tres aspectos: el error absoluto acumulado, el consumo total de energía y el número de muestras por segundo. El controlador PID-DGP presentó un mejor desempeño con respecto al control PID-NL en los primeros dos aspectos y es más lento en velocidad de procesamiento, como resultado final de este análisis experimental, se observó que al agregar ganancias variables a la estructura del control PID, se obtuvo una mejora significativa del sistema en lazo cerrado.

En otras palabras, al introducir ganancias variables al controlador PID, se obtuvo un controlador robusto ante cambios en el puntos de operación, pero al utilizar ganancias programables difusas el desempeño resulta ser mucho mejor. Como trabajo futuro se va a realizar una comparación experimental con los mismos controladores, para el caso de seguimiento de trayectoria. Adicionalmente se pretende incluir un sensor de corriente para calcular el par torsional del MCDIP y estimar la potencia eléctrica consumida en cada experimento.

\section{Referencias}

1. Armstrong, B., Neevel, D., Kusik, T.: New results in npid control: tracking, integral control, friction compensation and experimental results. IEEE Trans. Control Syst. Technol. 9, 399406 (2001)

2. Armstrong, B., Wade, B.: Nonlinear pid control with partial state knowledge: damping without derivatives. Int. J. Robotics Research 19, 715-731 (2000)

3. Aydogdu, O., Korkmaz, M.: A simple approach to design of variable parameter nonlinear pid controller. In: International Conference on Electrical Engineering and Applications, Chennai, India. pp. 81-85 (2011)

4. Cerecero-Natale, L.F., Ramos-Fernández, J.C., Marquéz-Vera, M., Campos-Mercado, E.: Control pi difuso de ganancias programables para un sistema mecatrónico de posicionamiento angular-lineal. Research in Computing Science 116, 157-169 (2016)

5. Chen, W., abd P.J. Gawthrop, D.B., Gribble, J., O'Reilly, J.: Nonlinear pid predictive controller. IEEE Proc. Control Theory Appl. 146, 603-611 (1999)

6. El-samahy, A.A., Shamseldin, M.A.: Brushless dc motor tracking control using self-tuning fuzzy pid control and model reference adaptive control. Ain Shams Engineering Journal (2016)

7. Graffin, I.: On-line PID controller tuning using Genetic Algorithms. DCU (2003)

8. Gu, J., Zhang, Y., Gao, D.: Application of nonlinear pid controller in main steam temperature control. In: Asia Pacific Power and Energy Engineering Conference, Wuhan, China. pp. 1-5 (2009)

9. Han, J.: Nonlinear pid controller. Acta Automatica Sinica 20, 487-490 (1994)

10. Khin, H.N., Che, F.Y., Eileen Lee, M.S., Liang, X.W.: Alpha beta gamma filter for cascaded pid motor position control. Engineering Procedia 41, 244-250 (2012)

11. Lin, P., Hwang, S., Chou, J.: Comparison on fuzzy logic and pid controls for a dc motor position controller. Industry Applications Society Annual Meeting,Conference Record of the IEEE 3, 1930-1935 (1994)

12. Vasu, N., Srinivasulu, G.: Comparative study of fuzzy pd controller and conventional controllers. Intelligent Agent and Multi-Agent Systems, IAMA, July pp. 22-24 (2009) 
Comparación experimental de controladores PID clásico, PID no lineal y PID difuso ...

13. Visioli, A.: Optimal tuning of pid controllers for integral and unstable processes. Proceedings of the IEEE, Part D 148(2), 180-194 (2001)

14. Ying, W., Hang, J., Min, Z.: The research on fuzzy pid control of the permanent magnet linear synchronous motor. Physics Procedia 24, 1311-1318 (2012)

15. Zaki, A.M., El-Bardini, Mohammad, S.F.S.M.M.: Embedded two level direct adaptive fuzzy controller for dc motor speed control. Ain Shams Engineering Journal (2015)

16. Zhen-Yu, Z., Masayoshi, T., Satoru, I.: Fuzzy gain scheduling of pid controllers. IEEE transactions on systems, man, and cybernetics 23, 1392-1398 (1993) 